\title{
EFEITO DO PRÉ-RECOBRIMENTO DE MICROPARTÍCULAS DE DIAMANTE NA ADESÃO E DISPERSÃO DA CARGA EM FORMULAÇÃO DE COMPÓSITOS COM MATRIZ EPÓXI/POLIAMIDA*
}

\author{
Magno Luiz Tavares Bessa ${ }^{1}$ \\ Rubén Jesus Sánchez Rodríguez ${ }^{2}$ \\ Ricardo Abrahão Júnior
}

\section{Resumo}

Visando melhorar a distribuição de micropartículas de diamante (MD) na formulação de compósitos, foi desenvolvida, através da emulsão do sistema epóxi/poliamida em água, uma técnica de encapsulamento de MD. As MD foram pré-recobertas com um polímero baseado no sistema epóxi-poliamida e posteriormente utilizadas como carga na produção de um compósito com matriz da mesma composição do revestimento. A matriz epóxi/poliamida e compósitos com carga de MD encapsulados e sem recobrimento foram avaliados. As propriedades mecânicas foram avaliadas por ensaio de flexão em 3 pontos e por análise de tenacidade à fratura e as propriedades térmicas por DMA. Por MEV foram observadas a microestrutura das MD encapsulados e a superfície de fratura dos corpos de prova após ensaio de flexão. Não foram observados aumentos nas propriedades mecânicas do compósito com carga MD encapsulados em relação ao compósito com carga de MD não modificados. Esse resultado foi devido à adição ao sistema de defeitos estruturais como bolhas e ar e porosidade provenientes do uso das MD encapsulados. Foi observado porém, melhor distribuição das MD no sistema e uma melhor adesão à matriz para a carga de $\mathrm{MD}$ encapsulados em relação à carga de $\mathrm{MD}$ não revestidos, em função dos resultados obtidos por DMA.

Palavras-chave: Resina epóxi, Micropartículas de diamante, Dispersão, Revestimento da carga

\section{EFFECT OF PRE-COATING OF DIAMOND MICROPARTICLES IN ADHESION AND DISPERSION OF FILLER IN EPOXY/POLYAMIDE MATRIX COMPOSITE FORMULATION Abstract}

In order to improve the distribution of diamond microparticles (MD) in the composite formulation, an MD encapsulation technique was developed through the epoxy/polyamide in water emulsion. The MD were precoated with a polymer based on the epoxy-polyamide system and subsequently used as filler in the production of a matrix composite of the same coating composition. The epoxy / polyamide matrix and composites with encapsulated and uncoated MD filler were evaluated. The mechanical properties were evaluated by a 3-point bending test and by fracture toughness analysis and thermal properties by DMA. By SEM the microstructure of the encapsulated MD and the fracture surface of the specimens after bending test were observed. No increases were observed in the mechanical properties of the composite with MD charge encapsulated in relation to unmodified MD filler composite. This result was due to the addition to the system of structural defects such as bubbles and air and porosity from the use of encapsulated MDs. It was observed, however, a better distribution of $M D$ in the system and a better adhesion to the matrix for the filler of MD encapsulated in relation to the filler of uncoated MD, in function of the results obtained by DMA.

Keywords: Epoxy Resin; Diamond Microparticles; Dispersion; Filler Coating.

1 Engenheiro Metalúrgico, Mestre, Doutorando no programa de Pós-graduação em Engenharia e Ciências dos Materiais, Setor de Polímeros, Universidade Estadual do Norte Fluminense, Campos dos Goytacazes, Rio de Janeiro, Brasil.

2 Doutorado em Ciências e Tecnologia de Polímeros, Professor Titular, Setor de Polímeros, Universidade Estadual do Norte Fluminense, Campos dos Goytacazes, Rio de Janeiro, Brasil.

3 Graduando em Engenharia Metalúrgica, Iniciação Científica, Setor de Polímeros, Universidade Estadual do Norte Fluminense, Campos dos Goytacazes, Rio de Janeiro, Brasil. 


\section{INTRODUÇÃO}

Materiais compósitos com matriz de resina epóxi Diglicidil Éter de Bisfenol A (DEBGA) e carga de micropartículas de diamante tem sido formulados [1,2]. Para esses casos, os estudos indicam que os impactos da introdução de micropartículas de diamante são fortemente dependentes, além da estrutura da rede epóxi, da dispersão dessa carga na matriz, onde a presença de agregados tem sido um fator negativo em relação as propriedades mecânicas [1,2].

Quanto menor o tamanho das partículas, maior será a contribuição da interfase nas propriedades, o que pode trazer benefícios às propriedades dos compósitos; porém a tendência de aglomeração entre as partículas pode prejudicar a melhora destas propriedades [3].

Para um efetivo incremento nas propriedades dos compósitos, é necessária a dispersão dos aglomerados e a obtenção de distribuição homogênea das partículas na matriz [4], capacidade que está limitada pela presença de intensas forças de Van Der Waals entre as partículas de tamanho nano-micrométrico.

O objetivo deste trabalho foi avaliar o efeito do pré-recobrimento das micropartículas de diamante num processo de emulsão epóxi/poliamida-água com vista a controlar o processo de agregação na formulação dos compósitos em matriz epóxi.

\section{MATERIAIS E MÉTODOS}

\subsection{Materiais Utilizados}

a) Resina Epóxi do tipo diglicidil éter de bisfenol A (DGEBA) Fornecido por Dow Química S/A; nome comercial DER 331

Densidade: $1,16 \mathrm{~g} / \mathrm{mL}$, massa molar: $340,41 \mathrm{~g} / \mathrm{mol}$

b) Agente de cura epóxi composto por Poliamida reativa de média viscosidade Fornecido por America Sales Representative; nome comercial ARP-25 Densidade $0,98 \mathrm{~g} / \mathrm{mL}$

c) Tensoativo Tween 80 Fornecido por Vetec Densidade: $1,08 \mathrm{~g} / \mathrm{mL}$, massa molar: $1310 \mathrm{~g} / \mathrm{mol}$.

d) Micropartículas de diamantes sintéticos

Granulometria: 1,5-3,5 $\mu \mathrm{m}$

Produzidas no Setor de Materiais Superduros do Laboratório de Materiais Avançados da Universidade Estadual do Norte Fluminense LAMAV/UENF

\subsection{Métodos}

\subsubsection{Pré-encasulamento das MD com o sistema epóxi/poliamida}

As MD foram encapsuladas através da técnica de emulsão. Foi acrescentado à resina epóxi DGEBA o tensoativo Tween 80 em uma quantidade de aproximadamente $0,2 \%$ em relação a quantidade de MD do sistema, para favorecer a dispersão destes na resina. Foi acrescentado $20 \%$ de MD, que foram misturadas à resina DGEBA manualmente e levados ao ultrassom por 30 minutos. Posteriormente, foi acrescentado ao sistema o endurecedor Poliamida, na proporção estequiométrica endurecedor-resina, seguido por agitação mecânica à 450 rpm por 3 
minutos. A emulsão foi promovida após a adição de água na proporção de 6 partes de água para 4 partes de DGEBA/poliamida utilizando um agitador Ultra-Turrax à 12000 rpm por 3 minutos. O material foi transferido para um refratário de vidro onde secou em estufa à $80^{\circ} \mathrm{C}$ por $24 \mathrm{~h}$. Em seguida, foi promovida uma pós-cura em estufa à $110^{\circ} \mathrm{C}$ por $2 \mathrm{~h}$. Foi obtido um material sólido poroso, submetido a um processo de moagem em um moinho de alta energia, por 5 minutos, obtendo-se 0 particulado composto por MD encapsulados.

\subsubsection{Formulação dos compósitos}

Em ambos os sistemas compósitos, tanto o com adição de carga sem nenhum tratamento (DGEBA/PA-MD) quanto o com adição de MD encapsulados (DGEBA/PA-MD encap.), a carga, sob aquecimento à $70^{\circ} \mathrm{C}$, foi misturada a resina epóxi DGEBA para obter-se uma composição final de $5 \%$ de carga de MD. Após aplicação de uma pressão reduzida, para retirar eventuais bolhas de ar introduzidas no processo de mistura, foi adicionado o endurecedor poliamida, na proporção estequiométrica endurecedor-resina. Após isto, o material foi transferido para moldes metálicos, onde foi realizada a cura à temperatura ambiente por 24 horas e um posterior processo de pós-cura a $60^{\circ} \mathrm{C}$ por 4 horas, seguido de $120^{\circ} \mathrm{C}$ por 4 horas e, por fim, $190^{\circ} \mathrm{C}$ durante 2 horas.

\subsubsection{Caracterização Mecânica}

\subsubsection{Ensaio de flexão em três pontos}

Foram realizados ensaios de flexão no método de três pontos utilizando uma máquina universal de ensaios mecânicos, marca Instron, modelo 5582 (SMMA/LAMAV/UENF). Os valores de tensão de flexão $(\sigma)$ e módulo de flexão $(E)$ foram determinados. As medições foram realizadas utilizando uma velocidade de 1,7 $\mathrm{mm} / \mathrm{min}$, com suporte de comprimento igual a $50 \mathrm{~mm}$, célula de carga $100 \mathrm{KN}$ e amostras com dimensões aproximadas de $65 \times 12 \times 4 \mathrm{~mm}^{3}$, de acordo com a norma ASTM D 790-03 [5]. Foram feitas três réplicas dos corpos de prova. Os resultados foram calculados pelas seguintes Equações:

$$
\begin{aligned}
& \sigma=\frac{3 P L}{2 b d^{2}} \\
& E=\frac{L^{3} m}{4 b d^{3}}
\end{aligned}
$$

Onde: $\mathrm{P}$ é a carga, b e d são a largura e a espessura da amostra respectivamente, $\mathrm{L}$ é o comprimento entre apoios e $\mathrm{m}$ é o declive da parcela inicial em linha reta da curva de carga de deformação.

\subsubsection{Análise de tenacidade à fratura $\left(\mathrm{K}_{\mathrm{IC}}\right)$}

A propriedade de tenacidade à fratura consiste na habilidade de um determinado material resistir à fratura quando uma trinca está presente (resistência à propagação de trincas). Para a determinação de Kıc por flexão em um sistema de três pontos, de acordo com a norma ASTM D 5045 [6], foi induzido num corpo de prova com dimensões aproximadas de $65 \times 12 \times 4 \mathrm{~mm}^{3}$ uma trinca de $5,4 \mathrm{~mm}$. $\mathrm{O}$ 
ensaio foi realizado com uma velocidade de $1,7 \mathrm{~mm} / \mathrm{min}$. Foram testadas 3 amostras para cada sistema e a tenacidade à fratura foi determinada através da seguinte equação:

$$
K_{I C}=\frac{P_{\max }}{B W^{1 / 2}} f(a / w)
$$

Onde: $\mathrm{P}$ é a carga máxima da falha, $\mathrm{B}$ é a espessura da amostra, $\mathrm{W}$ é o comprimento total, "a" é o comprimento da trinca e $f(a / w)$ é a expressão usada de acordo com a geometria da amostra apresentada na norma ASTM D5045.

\subsubsection{Caracterização Térmica}

A análise dinâmico-mecânica foi realizada na unidade de caracterização térmica do SEPOL/LAMAV/UENF. Os ensaios foram realizados utilizando corpos de prova retangulares, com dimensões de aproximadamente $35 \times 12 \times 4 \mathrm{~mm}^{3} \mathrm{em}$ um DMA Q 800 multi-frequência da TA Instruments com módulo de resfriamento (GCA). A garra utilizada foi a de flexão em três pontos. As condições de análise utilizadas foram frequência de $1 \mathrm{~Hz}$, amplitude de $10 \mu \mathrm{m}$, força estática de $0,1 \mathrm{~N}$ e taxa de aquecimento de $2^{\circ} \mathrm{C} \min ^{-1}$. $\mathrm{O}$ módulo de armazenamento ( $E$ ') e tan $\delta=E ' / E$ ' foram obtidos a partir de 30 a $190^{\circ} \mathrm{C}$.

\subsubsection{Análise microestrutural}

As análises da microestrutura das amostras foram realizadas no microscópio eletrônico Shimadzu, modelo SSX-550, disponível no LAMAV/CCT/UENF e no microscópio Jeol, modelo JSM-6460 LV, disponível na COPPE/UFRJ. Para avaliar suas morfologia e tamanho, a análise por MEV se realizou em amostra de MD encapsulados e também nas MD não revestidos e, para se avaliar a dispersão das cargas de MD na formulação dos compósitos, também foi observada a superfície de fratura dos corpos de prova após ensaio de flexão.

\section{RESULTADOS E DISCUSSÃO}

\subsection{Morfologia das MD sem recobrimentos e MD encapsulados}

A observação por MEV das partículas de MD sem nenhum tratamento (Figura 1a) revelou sua morfologia, que se compreende em partículas angulares com superfícies relativamente planas, uma característica comum à partículas que possuem estruturas cristalinas. Fazendo-se uma estimativa do tamanho dessas MD (Figura 1b) observou-se uma variação aproximada de 1,5 $\mu \mathrm{m}$ até 3,5 $\mu \mathrm{m}$, mostrando uma dispersão pequena no tamanhos das partículas. Quanto a análise morfológica das partículas de MD recobertas através a técnica de emulsão observa-se a massa obtida por moagem após pré-recobrimento das micropartículas, formando agregados de tamanho entre 5-50 $\mu \mathrm{m}$ e formato irregular (Figura 2a). Esses agregados possuem sua morfologia formada por pequenas esferas de diamante encapsulado e partículas angulares associadas a diamantes sem recobrimento (Figura 2b). As imagens mostram a eficiência do processo na desagregação das partículas de MD, entretanto, também indica a existência de aglomeração de partículas recobertas numa massa de resina epóxi parcialmente curada com poliamida, o que exigiu o processo de moagem. Em consequência deste processo originou-se uma 
heterogeneidade, caracterizada por partículas encapsuladas e diamantes com recobrimento removido.
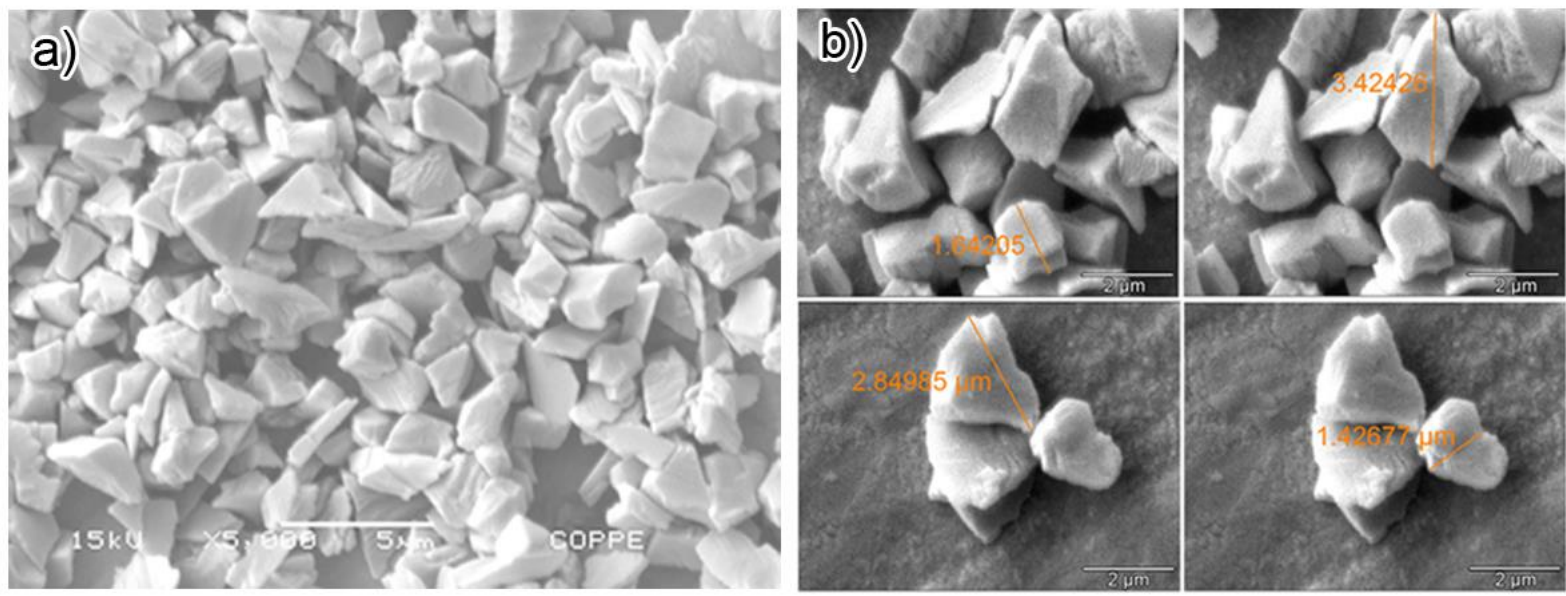

Figura 1. a) Morfologia das MD sem recobrimento - 5000X b) Estimativa de tamanho das MD
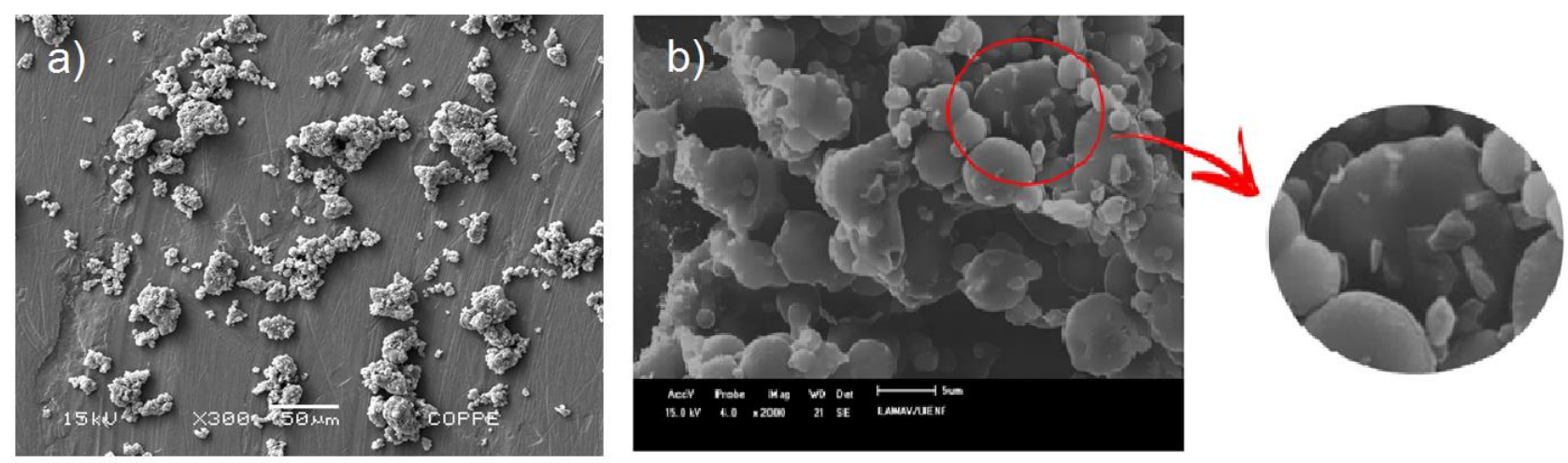

Figura 2. Morfologia dos MD encapsulados a) $300 \mathrm{X}$ e b) $2000 \mathrm{X}$

\subsection{Avaliação da dispersão de MD na formulação dos compósitos}

MEV foi utilizado para avaliar a dispersão das cargas de MD em formulações de compósitos com matriz DGEBA/PA. O processo de pré-recobrimento das MD favoreceu o processo de desaglomeração das MD, contribuindo para uma melhor dispersão destes na matriz epóxi. Para o compósito que se utilizou das MD sem nenhum tratamento é possível observar a presença de um aglomerado formado por de MD agregados (Figura 3a). Já para o compósito formulado com MD encapsulados observa-se um sistema mais homogêneo no que diz respeito à dispersão das MD na matriz. 

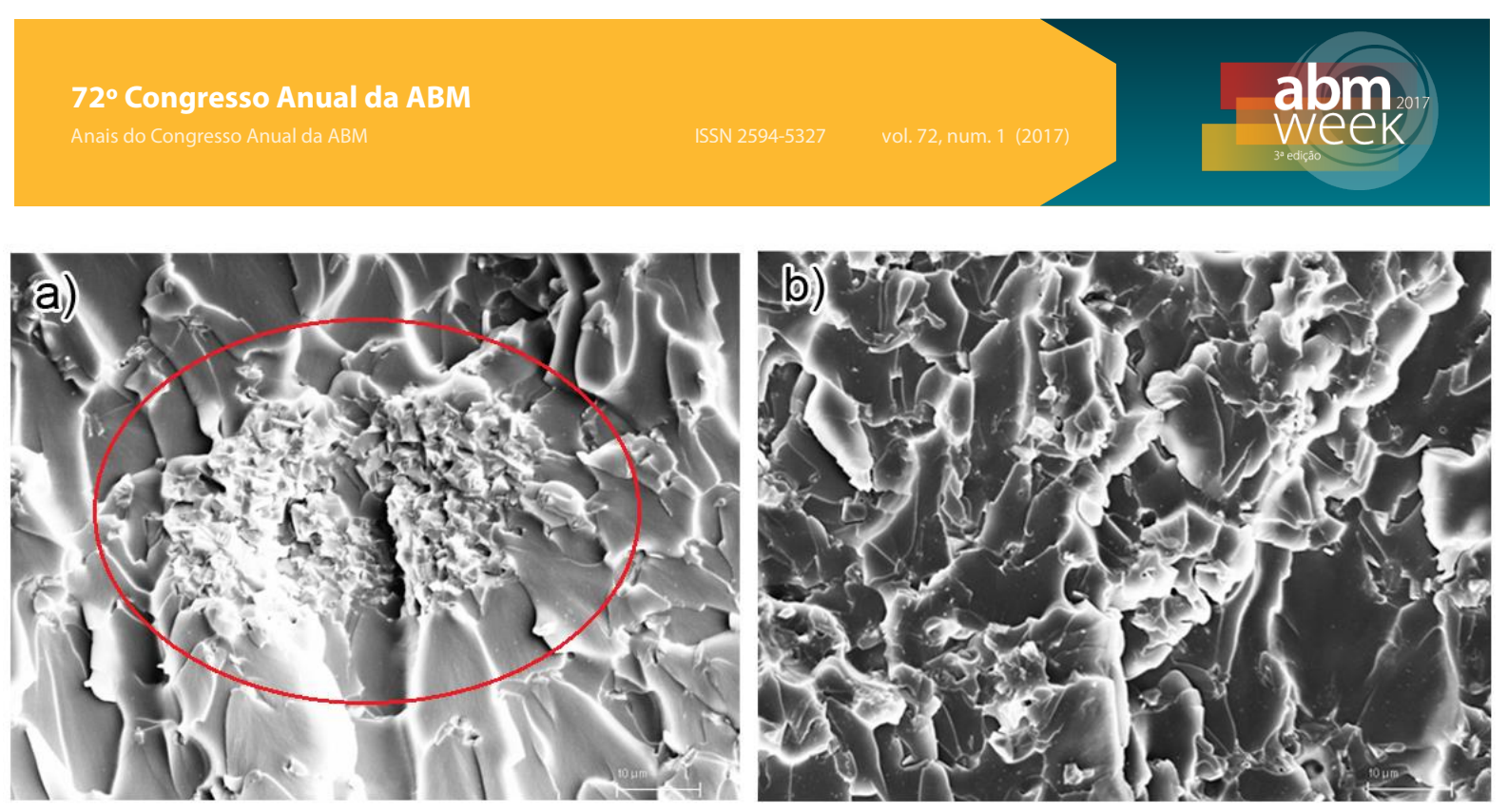

Figura 3. Superfície de fratura dos compósitos - 3080X a) DGEBA/PA-MD b) DGEBA/PA-MD encap.

\subsection{Propriedades Mecânicas}

Os módulos de elasticidade, tensões de ruptura e módulos de armazenamento para o sistema de matriz DGEBA/PA e para os sistemas compósitos DGEBA/PA-MD (sem revestimento) e DGEBA/PA-MD encap. (prérecoberto) são apresentados na Tabela 1.

Tabela 1. Propriedades mecânicas e térmica dos sistemas.

\begin{tabular}{lccc}
\hline Sistema & $\mathbf{E}(\mathrm{GPa})$ & $\boldsymbol{\sigma}_{\max }(\mathbf{M P a})$ & $\mathbf{E}^{\prime}(\mathrm{MPa})$ \\
\hline DGEBA/PA (matriz) & $2,49 \pm 0,15$ & $88,55 \pm 3,46$ & 1752 \\
\hline DGEBA/PA-MD & $2,83 \pm 0,11$ & $99,17 \pm 1,75$ & 1985 \\
\hline DGEBA/PA-MD encap. & $2,50 \pm 0,06$ & $884000 \pm 11,455$ & 1845
\end{tabular}

Os resultados não permitem observar um aumento no módulo de elasticidade e tensão de ruptura nos sistemas com carga de MD pré-revestidos quando comparados com a matriz sem adição de carga. O aumento da rigidez e resistência mecânica foi observado, de forma discreta, apenas no sistema com adição direta de carga, sem pré-encapsulamento.

A formulação com carga de MD revestidos, ainda que tenha favorecido a desagregação das $M D$, introduziu outros efeitos desfavoráveis no sistema, que foram os responsáveis pela baixo desempenho mecânico deste compósito. $\mathrm{Na}$ Figura 4 temos as superfícies de fratura dos compósitos formulados com MD sem tratamento e com MD pré-recobertos - Figura 4 a) e b), respectivamente. Para o sistema com MD sem tratamento observa-se uma microestrutura densa (Figura 4 a) enquanto o sistema com MD encapsulado revela uma microestrutura composta por pequenas cavidades, incorporadas ao sistema na utilização da carga formada pela massa moída obtida no processo de pré-recobrimento da MD pela técnica de emulsão. 

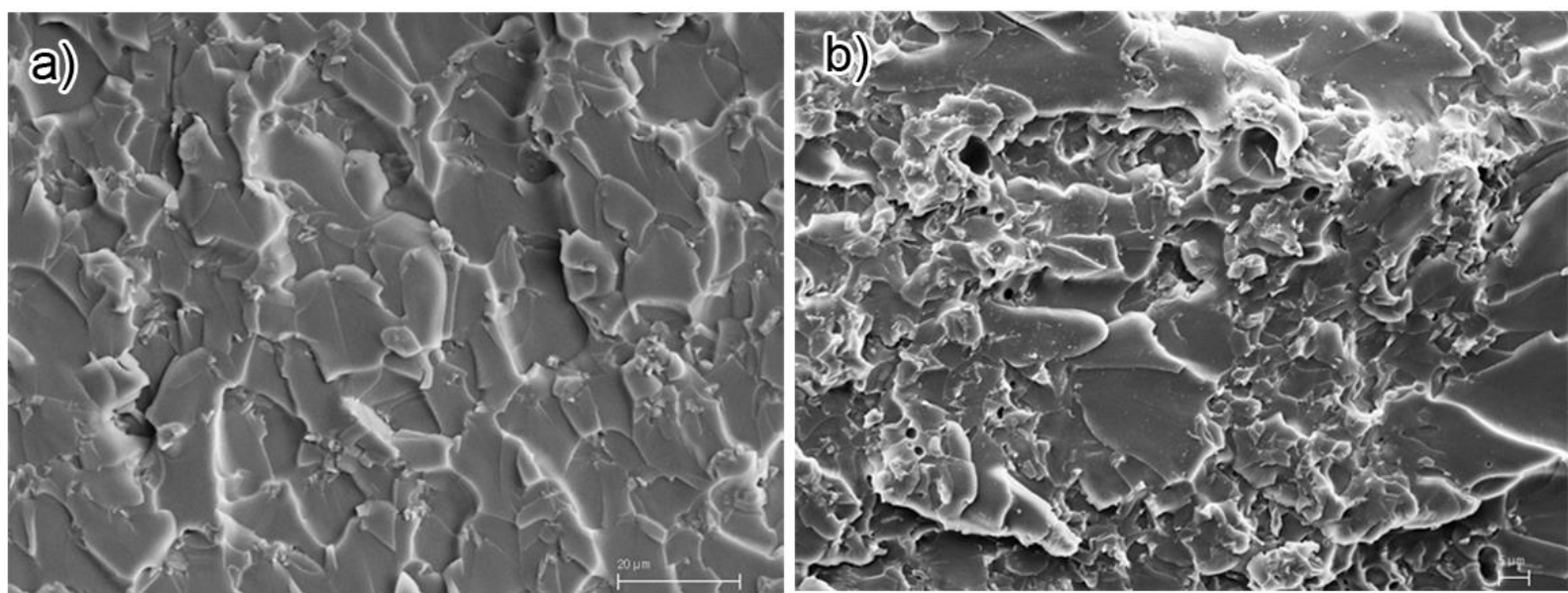

Figura 4. Superfície de fratura dos compósitos - 2120X a) DGEBA/PA-MD

b) DGEBA/PA-MD encap.

Um outro defeito estrutural responsável pelo menor desempenho mecânico do compósito formulado com MD pré-recobertos foi a presença de bolhas de ar aprisionadas no sistema, que agiram como concentradores de tensão (Figura 5 a) e que estão ausentes no sistema com MD sem tratamento (Figura 5 b). A alta viscosidade da formulação antes da cura dificultou a eliminação dessas bolhas de ar, que foram adicionadas durante o processo de mistura na matriz da carga de MD encapsulados. Esses efeitos estão relacionados à maior quantidade percentual de massa particulada adicionada à matriz polimérica para o sistema com carga prérevestida, em comparação ao sistema com adição de carga sem tratamento. A Tabela 2 evidencia a diferença na fração mássica para os sistemas.

Uma fração mássica 5 vezes maior para o sistema com carga revestida vem do pré-encapsulamento do diamante, uma vez que para se adicionar $5 \%$ de carga de diamante (como no sistema com diamante sem tratamento) foi necessário adicionar uma massa maior de particulado, sendo essa massa adicional referente ao material do recobrimento.

Tabela 2. Fração Mássica de particulado adicionado à matriz nos sistemas compósitos.

\begin{tabular}{lc}
\hline Sistemas & Fração Mássica de particulado adicionado à matriz \\
\hline DGEBA/PA-MD & 0,05 \\
\hline DGEBA/PA-MD encap. & 0,25 \\
\hline
\end{tabular}



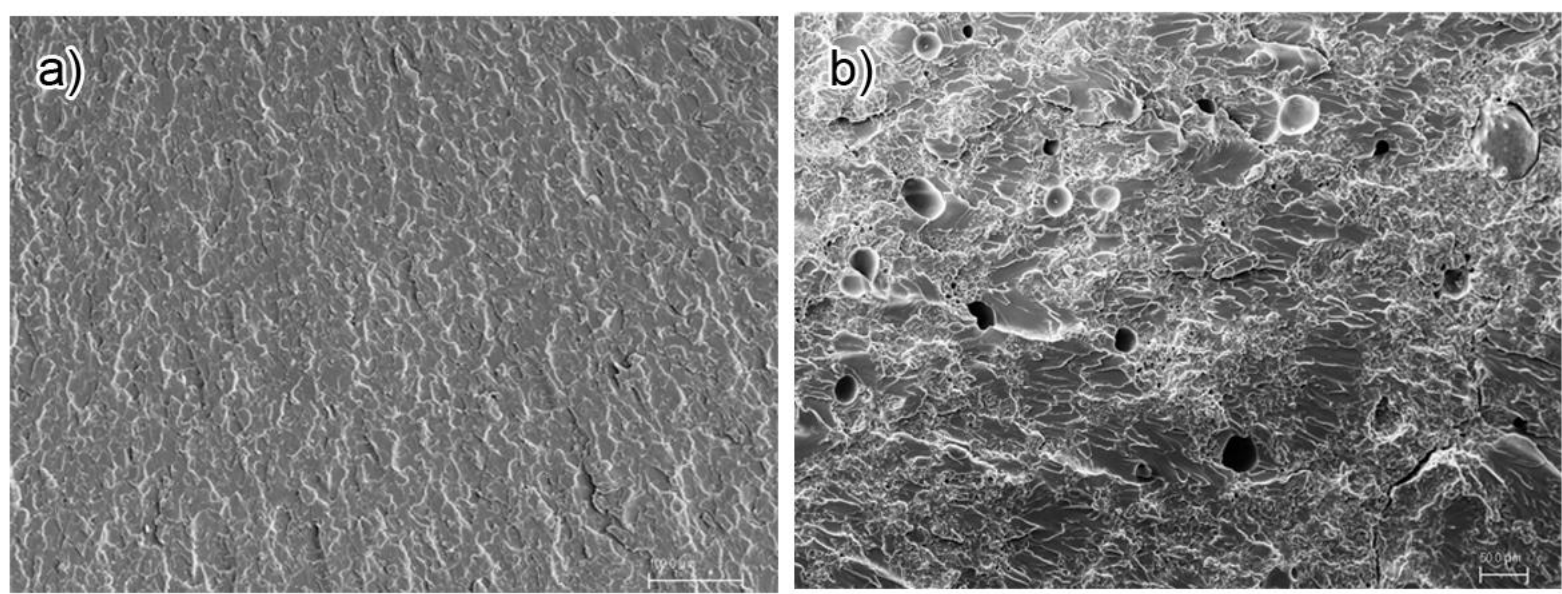

Figura 5. Superfície de fratura dos compósitos - 330X a) DGEBA/PA-MD b) DGEBA/PA-MD encap.

\subsection{Propriedades Térmicas}

Pelo ensaio de DMA foi possível obter as curvas Módulo de armazenamento (E') (Figura 6 a). Observou-se um menor valor de E' para o sistema com DGEBA/PAMD encap. quando comparado com o DGEBA/PA-MD (Tabela 1). Esse comportamento é similar ao encontrado para o módulo de elasticidade.

A análise das curvas de tan $\delta$ para todos os sistemas estudados (Figura 6 b) destaca o menor valor da altura do pico tan $\delta$ para o sistema com carga de MD encapsulados, em relação as outras formulações. Isto é um indicativo de que o revestimento da carga favoreceu a adesão da carga de diamante à matriz. Contrariamente, a existência de uma interfase microdiamante-resina fraca origina uma maior perda devido ao atrito interno entre as fases com menor adesão e consequentemente uma maior intensidade da tan $\delta[7]$.

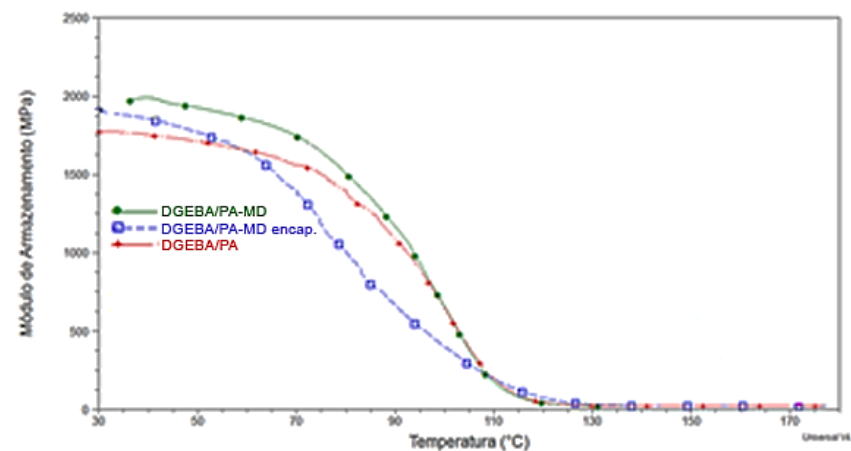

a)

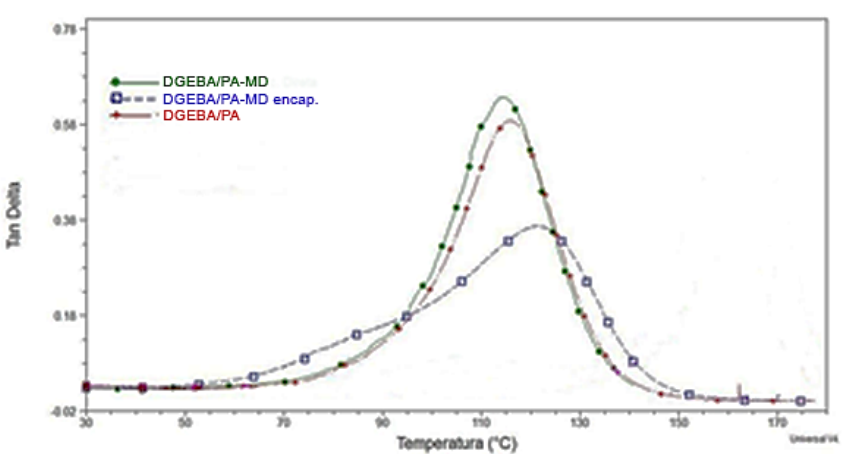

b)

Figura 6. a) Curvas Módulo de armazenamento versus Temperatura b) Curvas tan $\delta$ versus temperatura

A melhor adesão da carga para o sistema com MD encapsulados é um indicativo de que o pré-recobrimento é favorável em relação as propriedades mecânicas do sistema. Entretanto, o procedimento utilizado para a formulação do compósito, partindo da moagem das partículas recobertas, introduz heterogeneidade no sistema e exposição de partículas previamente recobertas, originando contatos entre as partículas de carga, o qual compromete a efetividade desta última etapa do procedimento. 


\section{CONCLUSÃO}

O processo de pré-recobrimento das MD foi favorável no que diz respeito à melhora da dispersão e adesão da carga à matriz porém o método de produção, que consistiu em uma técnica de emulsão que exige posterior moagem para a obtenção do particulado, foi responsável por efeitos não favoráveis ao sistema. Nos sistemas com MD pré-recobertos, tanto a presença de porosidade quanto o aprisionamento de bolhas de ar, causado pela alta viscosidade do sistema antes da cura, foram responsáveis pelo baixo desempenho mecânico dessa formulação de compósito. Para a solução desses problemas, um método de encapsulamento que originasse uma camada de recobrimento mais fina e recobrisse as MD de forma individual, não necessitando de um posterior processo de moagem, seria mais adequado.

\section{Agradecimentos}

A CAPES, CNPQ e a FAPERJ pelo apoio financeiro.

\section{REFERÊNCIAS}

1 C. R. Amaral, Tese de Doutorado, Universidade Estadual do Norte Fluminense, 2014.

2 M. L. T. Bessa, R. J. S. Rodríguez, C. R. Amaral in Anais do $1^{\circ}$ Encontro de Encontro de Engenharia, Ciência dos Materiais e Inovação do estado do Rio de Janeiro, Nova Friburgo, 2015.

3 P. M. Ajayan; L. S. Schadler; P. V. Braun Nanocomposite Science and Technology. 1ed. Weinheim: Wiley, 2003.

4 H. A. Al-Turaif, Prog. Org. Coat. 2010, 69, 241.

5 American Society for Testing and Materials. Flexural Properties of Unreinforced and Plastics and Electrical Insulating Materials: ASTM D 790-03. 2003.

6 American Society for Testing and Materials - ASTM D5045: Standard Test Methods for Plane-Strain Fracture Toughness and Strain Energy Release Rate of Plastic Materials, (2007).

7 J. Kubát; M. Riodhal; M. Welander Journal of Applied Polymer Science. 1990, 39,1527-1539. 